\title{
Evolution of Virulence in Natural Populations of the Satellite RNA of Cucumber mosaic virus
}

\author{
Fernando Escriu, Aurora Fraile, and Fernando García-Arenal
}

Depto. de Biotecnología, E. T. S. I. Agrónomos, Universidad Politécnica de Madrid, 28040 Madrid, Spain.

Accepted for publication 21 January 2000.

\begin{abstract}
Escriu, F., Fraile, A., and García-Arenal, F. 2000. Evolution of virulence in natural populations of the satellite RNA of Cucumber mosaic virus. Phytopathology 90:480-485.

From 1986 to 1992, an epidemic of tomato necrosis caused by Cucumber mosaic virus (CMV) plus CMV satellite RNAs (satRNAs) occurred in eastern Spain. From 1989 onward, the frequency of tomato necrosis diminshed, and it almost completely disappeared after 1991. Analyses of plants infected with CMV and with CMV satRNA and of the phenotype (necrogenic or nonnecrogenic for tomato) induced by some CMV satRNA variants, showed that the disappearance of tomato necrosis was due to changes in the genetic composition of the satRNA population (i.e., to its evolution toward decreased virulence). Analysis of components of the fitness of satRNA variants, necrogenic or nonnecrogenic for tomato, showed

that necrogenic and nonnecrogenic variants did not differ in infectivity or in their accumulation level in tomato and that they represented the same fraction of encapsidated RNA. Other fitness components were positively correlated with the greater virulence of necrogenic variants, in that they were favored in mixed infections with nonnecrogenic variants and were more effectively passed into CMV progeny than were nonnecrogenic variants. On the other hand, necrogenic CMV satRNA variants caused a more pronounced depression in the accumulation of CMV than did nonnecrogenic variants, which could affect the efficiency of aphid transmission. Thus, the evolution of virulence in the CMV satRNA population can be explained by trade-offs between factors that determine virulence and factors that affect transmission, as predicted by theoretical models on the evolution of virulence in parasites.
\end{abstract}

Modern agriculture is continuously challenged by rapidly evolving pests and pathogens. One of the most important evolving properties of pathogens is virulence, the understanding of which, though currently rudimentary, is critical for the ability to deal with established and new infectious diseases (5). Population models based on theoretical considerations or experiments carried out under controlled conditions have been proposed to explain the evolution of virulence (20). Nevertheless, detailed analyses of virulence evolution in natural populations are scarce; perhaps the best example is the classical work by Fenner and colleagues on myxoma virus infecting rabbits in Australia (6). To our knowledge, no similar work has been reported for any plant-infecting virus.

Cucumber mosaic virus (CMV) is a plant virus with a singlestranded, tripartite RNA genome encapsidated in isometric particles. CMV is the helper virus (HV) for a small, single-stranded satellite RNA (CMV satRNA) that modulates the replication and pathogenicity of CMV and depends on CMV for its replication, encapsidation, and transmission. CMV infects a very large number of plant species, in which it mostly induces symptoms of mosaic and stunting and is efficiently transmitted by many aphid species in a nonpersistent manner $(8,25)$.

As is the case in most temperate regions of the world, CMV is endemic on vegetable crops in Spain (18). In the late 1980s and early 1990s, an epidemic of tomato necrosis spread on tomato crops in eastern Spain, caused by CMV strains that supported satRNAs. Different satRNA variants were found associated to field isolates of CMV that, in tomato, induced either a systemic necrosis or a curl-stunt symptom or did not modify the mosaic, stunt, and fern leaf symptoms induced by CMV itself (10). We have reported on the genetic structure and variability of the satRNA population (3) and on the factors that determine its high variability (2) and have

Corresponding author: F. García-Arenal; E-mail address: fga@bit.etsia.upm.es

Publication no. P-2000-0320-01R

(C) 2000 The American Phytopathological Society also shown that, in field conditions, CMV satRNA behaves as a hyperparasite spreading epidemically on the populations of CMV (1).

Since 1990, CMV-associated tomato necrosis has become less frequent in tomato fields, almost disappearing after 1992. This could be attributed either to the disappearance of CMV satRNAs or their evolution toward reduced virulence. In this article, we show that the decrease of tomato necrosis in the field was due to the evolution of the CMV satRNA populations toward decreased virulence, and we analyze which factors in the satRNA life cycle could explain this evolution. To our knowledge, this is the first analysis of the evolution of virulence in natural populations of a plant virus.

\section{MATERIALS AND METHODS}

CMV and CMV satRNA isolates. Tomato plants showing symptoms of virus infection were sampled between 1989 and 1994 in the tomato-growing areas of eastern Spain, between Barcelona in the north and Murcia in the south, in the region affected by the epidemic of tomato necrosis (10). Symptomatic plants were sampled so that the number of necrotic and nonnecrotic plants reflected their frequency in the field. We define a CMV isolate as the virus preparation derived from a single field-infected plant. Virus isolates could support more than one electrophorectic variant of CMV satRNA, as analyzed by semidenaturing polyacrylamide electrophoresis (3). CMV satRNA variants were named using the format $N / i j$, in which $j=$ the electrophoretic variant from CMV isolate $i$ obtained in year $N$ (3). The following Spanish field isolates of CMV were used as HVs: MAD T93/4, MAD Z94/1, VAL M95/437, BAR M95/458, and MUR M95/429 of genetic type 1; and BAR T93/7, BAR T93/47, BAR T94/104, BAR T94/108, and BAR T94/96 of genetic type 2 (1). Genetic types 1 and 2 are as described by Fraile et al. (7). FnyCMV (23) was also used as a HV. CMV and CMV satRNA isolates were maintained in tobacco or tomato plants in a greenhouse. CMV particles were purified from infected tissues as described by Lot et al. (17), and RNA was extracted by disrupting the particles in sodium dodecyl sulfate (SDS) and partitioning in phenolchloroform. 
Production of biologically active cDNA clones of CMV satRNA variants. Full-length cDNA clones of CMV satRNAs were obtained as by Aranda et al. (2) by reverse transcription and polymerase chain reaction (RT-PCR) amplification using the primers 5'GGAATTCCCGGGTCCTG3', with restriction sites for endonucleases EcoRI and SmaI, and having eight bases (underlined) complementary to the 3' end of all CMV satRNAs reported to date; and 5'GGAATTCTAATACGACTCACTATAGGTTTTGTTTG3', with an EcoRI site, a modified T7-RNA polymerase promoter, and $10 \mathrm{nu}-$ cleotides (underlined) identical to those at the $5^{\prime}$ end of all CMV satRNA variants reported. cDNA was hydrolyzed using EcoRI and $S m a \mathrm{I}$ and cloned in the vector pBS+; infectious RNA was transcribed with T7-RNA polymerase (26).

Biological assays and quantification of CMV and CMV satRNA. Biological assays were mostly done in tomato plants (cv. Rutgers). For most assays, RNA transcribed from CMV satRNA full-length cDNA clones was inoculated at $20 \mu \mathrm{g} / \mathrm{ml}$ with virion RNA of satellite-free $\mathrm{HV}$ at $100 \mu \mathrm{g} / \mathrm{ml}$ in $0.1 \mathrm{M} \mathrm{Na}_{2} \mathrm{HPO}_{4}$. For the assays of satRNA infectivity, satRNA transcripts at concentrations of $1,0.1$, and $0.01 \mu \mathrm{g} / \mathrm{ml}$ were inoculated with $10 \mu \mathrm{g}$ of HV RNA per ml. In all cases, $10 \mu \mathrm{l}$ of inoculum was applied to the cotyledons or to the first true leaf of young tomato plants.

Quantification of virus accumulation was done by enzyme-linked immunosorbent assay (ELISA) using polyclonal antibodies raised against Fny-CMV (29). Dilutions $(100 \mu \mathrm{l})$ at $1 / 100$ and $1 / 1,000$ of each virus preparation to be quantified were loaded on ELISA plates together with a series of standards $\left(10^{0.5}\right.$ to $10^{-2} \mu \mathrm{g}$, in a series of $1 / 10^{0.5}$ dilutions) of Fny-CMV particles.

Quantification of satRNA was done by dot-blot hybridization (26) in total nucleic acid extracts (22) of tomato leaves. Hybridization was carried out at $40^{\circ} \mathrm{C}$ with $5^{\prime},{ }^{32} \mathrm{P}$-labeled oligonucleotides complementary to, approximately, nucleotides 250 to 260 of satRNA variants necrogenic ( $5^{\prime}$ GCGTCATGACTCATA3') or nonnecrogenic (5'CGTCATCCACGATAC3') for tomato (sequences specific for each group of satellites are underlined). After hybridization with the CMV satRNA-specific primers, nylon membranes were rehybridized at $65^{\circ} \mathrm{C}$ with a cDNA probe (approximately 800 nucleotides long) complementary to barley $18 \mathrm{~S}$ rRNA (9), so as to have an RNA-loading control. For qualitative detection of CMV satRNA, hybridization was carried out at $65^{\circ} \mathrm{C}$ with a cRNA probe derived from a full-length clone of B2 satRNA (4). All hybridizations were done overnight in $6 \times \mathrm{SSC}(1 \times \mathrm{SSC}$ is $0.15 \mathrm{M} \mathrm{NaCl}$ plus $0.015 \mathrm{M}$ sodium citrate) per $5 \times$ Denhardt's mix per $0.1 \%$ SDS per yeast tRNA at $250 \mu \mathrm{g} / \mathrm{ml}$. After exposure of the X-ray film, quantification was done by densitometry using the 1.52 version of NIH Image package (W. Rasband; NIH, Research Service Branch, NIMH, Bethesda, MD) (15). In each dot-blot membrane, a 1/5 dilution series of standards for necrogenic and nonnecrogenic satRNAs was included, and samples were loaded at different dilutions to ensure that their densitometric value fell within the linear portion of the densitometric curve for the standards (Fig. 1).

Quantification of the fraction of satRNA in virion RNA was done by densitometry of ethidium bromide-stained agarose electrophoresis gels.

Statistical analyses. Comparison of frequency distributions was done by a $\mathrm{G}$ test of independence for $\mathrm{R} \times \mathrm{C}$ contingency tables (page 745 in literature citation 28). Regressions of satRNA infection versus satRNA concentrations were compared by analyses of covariance (pages 499 and 509 in literature citation 28). Multiple (page 242 in literature citation 28) or orthogonal (page 530 in literature citation 28) comparisons were used for analyses of satRNA accumulation and of $\mathrm{HV}$ accumulation. Comparisons were done using

\section{A Hibridization with non-necrogenic satRNA specific probe}
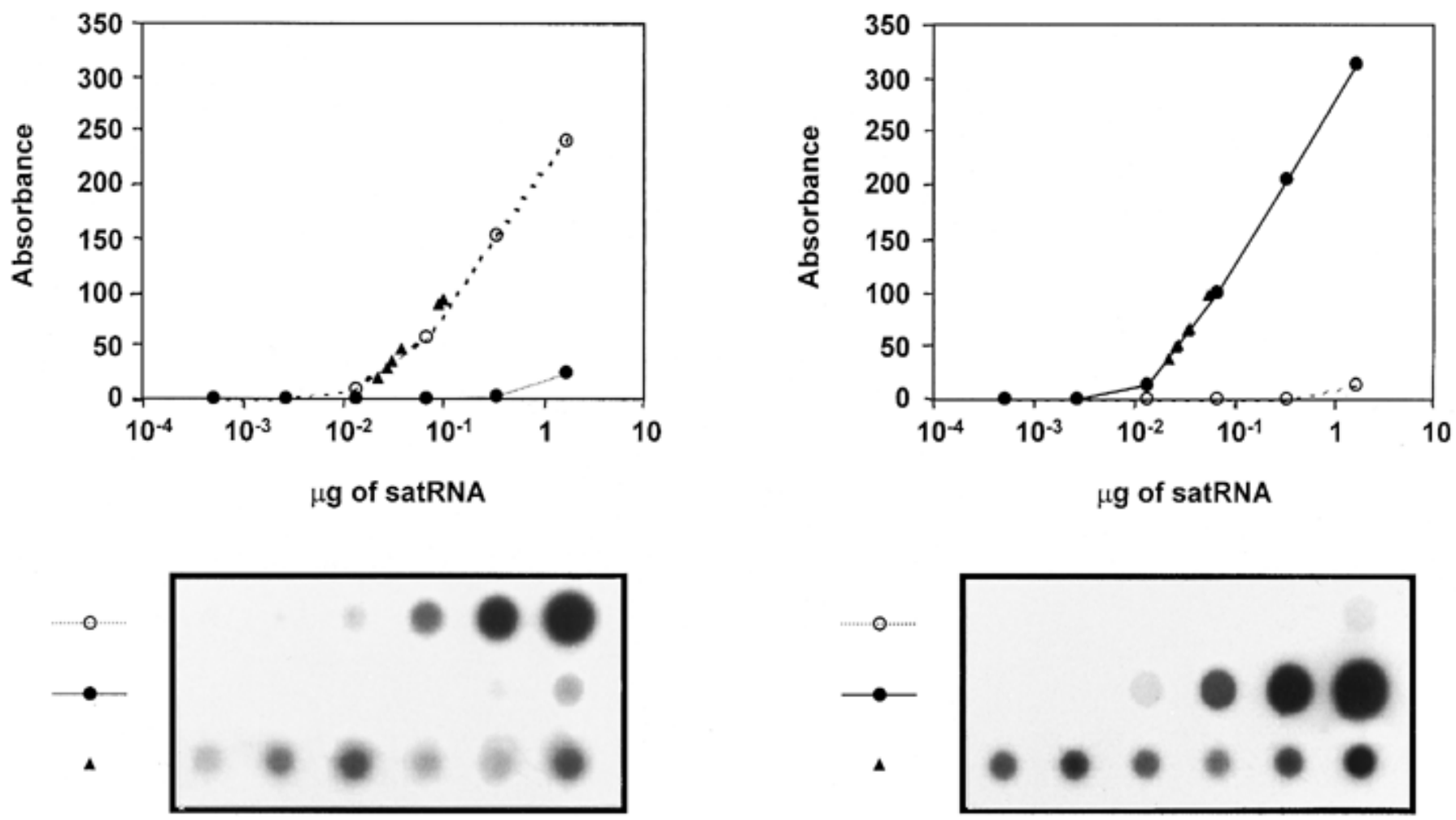

Fig. 1. Quantification of Cucumber mosaic virus satellite RNA (CMV satRNA) by dot-blot hibridization. Total nucleic acid extracts of six tomato plants infected by Fny-CMV and a mix of the necrogenic 91/5.1 and the nonnecrogenic 89/20.1 satRNA variants $(\boldsymbol{\Delta})$ were loaded together with necrogenic $(\bullet)$ and nonnecrogenic (O) CMV satRNA standards (2.7 ng to $1.7 \mu \mathrm{g}$, in a series of $1 / 5$ dilutions) and hibridized with a ${ }^{32} \mathrm{P}$-labeled oligonucleotide specific for $\mathbf{A}$, nonnecrogenic or B, necrogenic satRNA detection. For quantification, $25-\mu 1$ of $1 / 100$ and $1 / 1$ dilutions of extracts were used, so that absorbance values of samples fell within the linear portion of the standard's curves. Absorbances were determined by densitometry of blots and plotted against satRNA amount of standards. 
the Bonferroni and Kruskal-Wallis tests (pages 242 and 430 in literature citation 28). Data on accumulation of CMV particles were previously analyzed by a one-way analysis of variance after logarithmic transformation of the data for normality. All analyses were done using the Statgraphics Plus package (version 3.0; Statistical Graphics Corporation, Englewood Cliffs, NJ).

\section{RESULTS}

Populations of CMV satRNA evolve to reduced virulence. Two hundred eight tomato plants, sampled from 1989 to 1994, were analyzed for the presence of CMV and CMV satRNA (Table 1). Approximately three quarters of the plants were infected with CMV, in frequencies that varied from year to year. Symptomatic plants negative for CMV were mostly infected with Tomato spotted wilt virus (data not shown). The frequency of CMV-infected plants that harbored CMV satRNA increased from 1989 until 1990 to 1991, and diminished afterward. The relative increase of CMV satRNA frequency in 1994 is somewhat misleading, because all plants infected by CMV+satRNA came from a small area where tomato necrosis had reappeared in some fields. The frequency of CMV-infected plants showing necrosis diminished after 1990 and disappeared after 1991, with the above-mentioned exception in 1994. Because of the increase in frequency of plants infected with CMV+satRNA between 1989 and 1991, the decrease in frequency of necrosis is more evident if frequencies are estimated over plants infected with CMV+ satRNA. This also showed that the increase in frequency of CMV+ satRNA infections is not paralleled by an increase of tomato necrosis. The average number of electrophoretic variants of CMV satRNA per CMV+satRNA-infected plant is shown in Table 1; this value decreased from 1.75 in 1989 to 1.0 in 1994. This decrease paralleled that of tomato necrosis between 1989 and 1991.

CMV satRNA variants were randomly chosen for cDNA cloning. Full-length clones yielding infectious transcripts were obtained for 54 of the 60 chosen variants. The phenotype of these 54 variants was assayed on Rutgers tomato with Fny-CMV as a HV. Of these, 5 variants $(9.2 \%)$ did not modify the mosaic, stunt, and fern leaf symptoms caused by Fny-CMV; 33 variants (61.1\%) caused a symptom of leaf curl with extreme reduction of internode elongation

TABLE 1. Details of the Cucumber mosaic virus satellite RNA (CMV satRNA) populations and evolution of tomato necrosis in the field

\begin{tabular}{lcrrrrr}
\hline & \multirow{2}{*}{$\begin{array}{c}\text { No. of } \\
\text { plants }\end{array}$} & \multicolumn{2}{c}{$\begin{array}{c}\text { No. of } \\
\text { plants with }\end{array}$} & \multicolumn{2}{c}{ No. of plants with CMV satRNA } & \multirow{2}{*}{$\begin{array}{c}\text { satRNA } \\
\text { variants }\end{array}$} \\
\cline { 5 - 6 } Year & sampled & CMV & \multicolumn{1}{c}{ Total $^{\mathrm{y}}$} & Necrotic $^{\mathrm{z}}$ & Nonnecrotic $^{\mathrm{z}}$ & per plant \\
\hline 1989 & 27 & 27 & $18(0.67)$ & $14(0.78)$ & $4(0.22)$ & 1.75 \\
1990 & 22 & 22 & $21(0.95)$ & $12(0.57)$ & $9(0.43)$ & 1.60 \\
1991 & 21 & 11 & $9(0.82)$ & $4(0.44)$ & $5(0.56)$ & 1.33 \\
1992 & 36 & 36 & $17(0.47)$ & $0(0.00)$ & $17(1.00)$ & 1.37 \\
1993 & 40 & 33 & $12(0.36)$ & $0(0.00)$ & $12(1.00)$ & 1.43 \\
1994 & 62 & 23 & $14(0.61)$ & $7(0.50)$ & $7(0.50)$ & 1.00 \\
Total & 208 & 152 & $91(0.60)$ & $37(0.41)$ & $54(0.59)$ & 1.50
\end{tabular}

y Data in parentheses are frequency of plants with CMV satRNA over plants with CMV.

${ }^{z}$ Data in parentheses are frequencies of necrotic or nonnecrotic plants over total of plants with CMV satRNA.

TABLE 2. Phenotype of cloned Cucumber mosaic virus satellite RNA (CMV satRNA) variants as related to the symptoms of the field plants from which they were isolated ${ }^{y}$

\begin{tabular}{lccc}
\hline & & \multicolumn{2}{c}{ Symptom of original field plant } \\
\cline { 3 - 4 } Phenotype $^{\mathrm{z}}$ & Total & Necrosis & Nonnecrosis \\
\hline Necrosis & 16 & 13 & 3 \\
Curl stunt & 33 & 12 & 21 \\
Fern leaf & 5 & 4 & 1 \\
Total & 54 & 29 & 25 \\
\hline
\end{tabular}

y Data are number of satRNA variants.

${ }^{\text {z }}$ CMV satRNA variants were assayed on tomato with Fny-CMV as a helper virus. (curl-stunt symptom); and 16 variants $(29.7 \%)$ caused a necrosis. Of these, 14 caused a systemic necrosis initially associated to vascular tissues typical of CMV satRNAs necrogenic for tomato and 2 caused a systemic necrosis that was initiated as necrotic spots in the leaf lamina. To examine the possibility that the phenotype of the assayed CMV satRNA variants varied according to the strain of HV, a second assay was done in which Rutgers plants were inoculated with five randomly chosen CMV satRNA variants that were necrogenic in the previous assay (89/15.1, 89/42.1, 90/22.1, 91/5.1, and 94/32.1) and five randomly chosen nonnecrogenic CMV satRNA variants $(89 / 15.2,89 / 20.1,90 / 17.1,90 / 19.2$, and 91/2.2). Each of the 10 variants was assayed with 10 field isolates of CMV, five from genetic type 1 and five from genetic type 2, the two genetic types most prevalent in Spanish populations of CMV (7). In all instances, the satRNA variants that were necrogenic with FnyCMV were necrogenic for tomato with all 10 field CMV isolates, and no CMV satRNA variant that was nonnecrogenic with FnyCMV caused necrosis with any of the 10 field CMV isolates. No effect of the HV on the expression of the satRNA-induced necrosis was observed. Thus, the ability to induce necrosis in tomato appeared to depend on the CMV satRNA and not on the interaction of CMV satRNA variants and the CMV isolate.

The phenotype of the 54 cloned CMV satRNA variants, when assayed with Fny-CMV as a HV, in relation to the symptoms shown by the field tomato plant from which they were isolated, is shown in Table 2. The type of symptom (necrosis or nonnecrosis) shown by the original field plant and the phenotype (necrogenic or nonnecrogenic) shown by the satRNAs with Fny-CMV as a HV were statistically dependent ( $P=0.007$ in test of independence). Most ( 0.81$)$ necrogenic satRNAs came from plants with symptoms of systemic necrosis. A smaller fraction of nonnecrogenic satRNA variants $(0.58)$ came from nonnecrotic field plants. Mixed infections of necrogenic and nonnecrogenic satRNAs were more frequent in necrotic (0.55) than in nonnecrotic (0.12) field tomato plants (Table 2), which suggests that, in mixed infections, the necrogenic phenotype is dominant over the nonnecrogenic. It could be that these results were biased by RT-PCR-introduced mutations, because it has been shown that a single replacement at one of the positions that determine the necro-

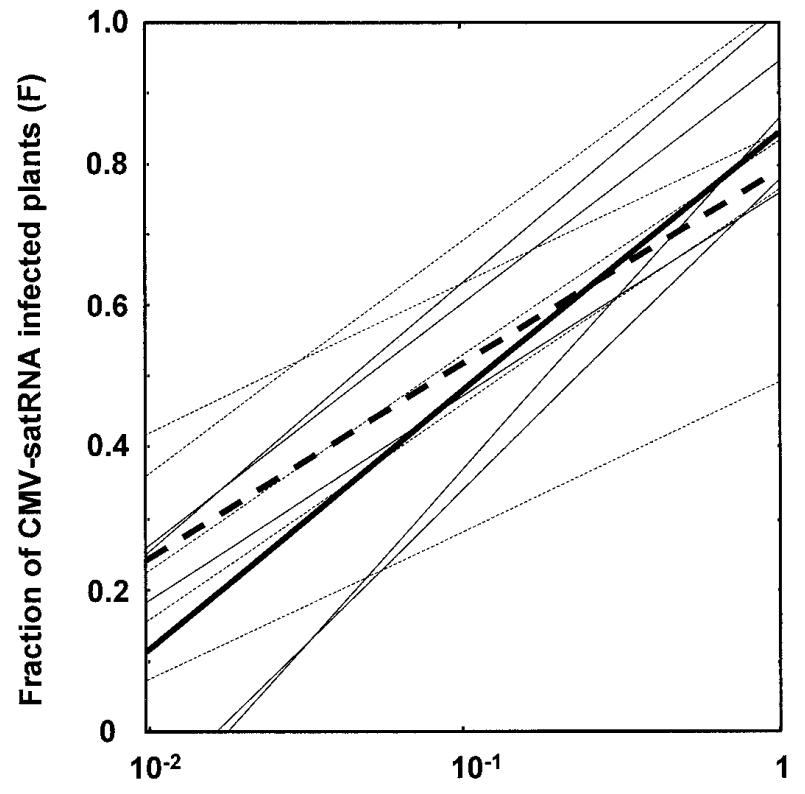

SatRNA concentration (C)

Fig. 2. Regression lines of the fraction of Cucumber mosaic virus satellite RNA (CMV satRNA)-infected plants $(\mathrm{F})$ versus satRNA concentration $(\mu \mathrm{g} / \mathrm{ml})$ (C) in the inoculum for five necrogenic (thin solid line) or nonnecrogenic (thin dashed line) satRNA variants and for all necrogenic (thick solid line) or nonnecrogenic (thick dashed line) variants pooled together. 
genic or nonnecrogenic phenotype of satRNA for tomato is sufficient to change its phenotype (24). By sequencing independently obtained duplicate clones of 15 satRNA variants, in our hands, the RTPCR error rate was estimated to be $2.35 \cdot 10^{-3}$ misincorporations per nucleotide. Thus, the probability of a misincorporation occurring at one of the bases that determine necrogenicity would be $2.10 \cdot 10^{-5}$.

To test the hypothesis of dominance for the necrogenic phenotype, 20 random combinations of 10 necrogenic $(89 / 15.1,89 / 42.1$, 89/42.4, 90/5.1, 90/22.1, 90/24.1, 91/3.1, 91/3.2, 91/5.1, and 94/32.1) and 10 nonnecrogenic $(89 / 15.2,89 / 20.1,89 / 23.1,90 / 13.1,90 / 14.1$, 90/17.1, 90/19.2, 91/2.2, 92/4.1, and 92/10.1) CMV satRNA variants, each with one necrogenic and one nonnecrogenic, were inoculated with Fny-CMV as a HV on 10 plants of Rutgers tomato each. For 18 combinations, all 10 plants per treatment became necrotic; for the other two combinations, $1 / 10$ and $0 / 10$ plants became necrotic. Both of these two combinations included the necrogenic satRNA 91/3.1, which was isolated from a nonnecrotic field plant. On the other hand, the combination involving satRNA 91/3.2, isolated from the same field plant, resulted in the necrosis of the 10 assayed plants. The results indicate that, in most instances, necrosis is dominant over nonnecrosis in mixed infections.

All of these results taken together indicate that the decrease in frequency of tomato necrosis observed in the field is due to a decrease in the frequency of necrogenic CMV satRNAs in the satRNA field population and, consequently, reflects the evolution of the CMV satRNA population toward reduced virulence. Thus, under conditions of natural infection of tomato, CMV satRNA necrogenic for tomato should be at a disadvantage (i.e., less fit) as compared with nonnecrogenic forms.

Analyses of fitness components in necrogenic and nonnecrogenic variants of CMV satRNA. A series of factors in the life cycle of the satRNA that could influence its fitness was analyzed. These factors were infectivity, accumulation in single and mixed infections, encapsidation, and probability of passage into the descent of CMV.

TABLE 3. Accumulation of Cucumber mosaic virus satellite RNA (CMV satRNA) in tomato leaves ${ }^{y}$

\begin{tabular}{llc}
\hline satRNA variant & Single infection & Mixed infection \\
\hline Necrogenic & & \\
$89 / 15.1$ & $40.39 \pm 8.16$ & $27.03 \pm 3.84$ \\
$89 / 42.1$ & $22.31 \pm 3.41$ & $16.97 \pm 1.58$ \\
$89 / 42.4$ & $29.41 \pm 4.60$ & $34.05 \pm 5.06$ \\
$90 / 5.1$ & $33.14 \pm 6.83$ & $13.99 \pm 1.79$ \\
$90 / 22.1$ & $30.48 \pm 4.09$ & $27.36 \pm 2.58$ \\
$90 / 24.1$ & $37.68 \pm 4.73$ & $21.96 \pm 5.26$ \\
$91 / 3.1$ & $29.77 \pm 6.12$ & nd \\
$91 / 3.2$ & $39.65 \pm 7.76$ & $52.16 \pm 8.17$ \\
$91 / 5.1$ & $47.58 \pm 8.50$ & $55.10 \pm 6.62$ \\
$94 / 32.1$ & $31.25 \pm 6.43$ & $52.36 \pm 6.44$ \\
$\bar{x} \pm s^{z}$ & $34.93 \pm 2.17 \mathrm{a}$ & $31.85 \pm 1.97 \mathrm{a}$ \\
Nonnecrogenic & & \\
$89 / 15.2$ & $10.41 \pm 3.96$ & $1.00 \pm 0.75$ \\
$89 / 20.1$ & $43.29 \pm 7.20$ & $0.91 \pm 0.43$ \\
$89 / 23.1$ & $22.84 \pm 8.72$ & $0.71 \pm 0.40$ \\
$90 / 13.1$ & $10.24 \pm 4.44$ & $8.70 \pm 3.02$ \\
$90 / 14.1$ & $29.26 \pm 5.15$ & $1.46 \pm 0.77$ \\
$90 / 17.1$ & $81.94 \pm 26.23$ & $0.74 \pm 0.39$ \\
$90 / 19.2$ & $29.74 \pm 11.31$ & $0.32 \pm 0.05$ \\
$91 / 2.2$ & $14.77 \pm 4.02$ & $4.94 \pm 1.33$ \\
$92 / 4.1$ & $23.11 \pm 1.38$ & nd \\
$92 / 10.1$ & $45.87 \pm 20.33$ & nd \\
$\bar{x} \pm s^{z}$ & $32.08 \pm 0.20 \mathrm{a}$ & $2.23 \pm 0.44 \mathrm{~b}$ \\
\hline
\end{tabular}

${ }^{y}$ Data are micrograms of satRNA per gram fresh weight in nucleic acid extracts of the third leaf above the inoculated one, when each satRNA variant was inoculated alone (single infection: mean of 10 plants \pm standard error) or in combination with each of two satRNA variants of the other type (mixed infection: mean of 20 plants \pm standard error, 10 plants in each combination). $\mathrm{nd}=$ Not determined.

${ }^{\mathrm{z}}$ Mean \pm standard error for all necrogenic or nonnecrogenic CMV satRNA variants. The same letter indicates that there are no significant differences in satRNA accumulation in a Bonferroni test.
The infectivity of 10 necrogenic and 10 nonnecrogenic CMV satRNA variants listed above was analyzed by monitoring the fraction of plants infected by the satRNA when inoculated at three concentrations of satRNA supported by the same concentration of FnyCMV. The regression of the fraction of plants infected versus satRNA concentration for some of the satRNA variants, and for the necrogenic or nonnecrogenic ones pooled together, is shown in Figure 2. Although differences in infectivity were found among individual satRNA variants, no significant differences were found between the necrogenic and nonnecrogenic variants (regression lines were $F=0.37 C+0.85$ and $F=0.28 C+0.80$, respectively, in which $F$ is the frequency of satRNA-infected plants and $C$ is the logarithm of satRNA concentration in the inoculum) when considered as a whole.

Accumulation in systemically infected leaves of Rutgers tomato was also assayed for the same set of 10 necrogenic and 10 nonnecrogenic CMV satRNA variants when supported by Fny-CMV. CMV satRNA accumulation was analyzed in single infections and in 20 mixed infections, in which each necrogenic or nonnecrogenic variant was assayed in combination with two nonnecrogenic or necrogenic variants, respectively. For each of the 40 treatments, satRNA accumulation was quantified in nucleic acid extracts of 10 plants (Table 3 ). In single infections, accumulation of necrogenic and nonnecrogenic variants of CMV satRNA did not differ significantly. Accumulation data in mixed infection showed that the presence of nonnecrogenic satRNA variants did not affect the accumulation level of necrogenic variants, while the presence of necrogenic variants resulted in a 10 -fold depression of the accumulation of the nonnecrogenic variants $(P<0.0001)$.

CMV particles were purified for each of the single-infection treatments in the experiment before, and the relative proportion of satRNA in particles was quantified by densitometry after agarose-gel electrophoresis and ethidium bromide staining. The fraction of virion RNA represented by satRNA did not differ significantly for necrogenic $(0.34 \pm 0.007)$ and nonnecrogenic $(0.36 \pm 0.011)$ variants. The presence of necrogenic or nonnecrogenic satRNA variants affected differently the amount of CMV RNAs in virus particles. The fraction of virion RNA made out of RNA1 + RNA2 was bigger $(P=0.01)$ when Fny-CMV supported necrogenic variants $(0.19 \pm 0.005)$ than when it supported nonnecrogenic variants $(0.15 \pm 0.010)$, and the reverse was true for RNA4 $(0.21 \pm 0.004$ and $0.24 \pm 0.006)$. The fraction of virion RNA made out of RNA3 did not differ $(0.25 \pm$ 0.006 in both cases).

For CMV to initiate an infection, a set of at least three particles, encapsidating RNA1, RNA2, and RNA3, is needed. It is not known if CMV satRNA occurs in the virus together with CMV RNAs or if it is encapsidated by itself (16). Thus, it could be that the average fraction of CMV satRNA over total encapsidated RNA does not reflect differences in the probability of transmission of different satRNA variants. To estimate the probability of transmission, the above-described virus preparations from tomato plants infected

TABLE 4. Frequency of transmission of Cucumber mosaic virus satellite RNA (CMV satRNA) variants with CMV

\begin{tabular}{lc|cc}
\hline satRNA variant & $\begin{array}{c}\text { Frequency of satRNA- } \\
\text { infected plants }^{\mathrm{y}}\end{array}$ & satRNA variant & $\begin{array}{c}\text { Frequency of satRNA- } \\
\text { infected plants }\end{array}$ \\
\hline Necrogenic & & Nonnecrogenic & \\
$89 / 15.1$ & 0.75 & $89 / 15.2$ & 0.10 \\
$89 / 42.1$ & 0.46 & $89 / 20.1$ & 0.70 \\
$89 / 42.4$ & 0.90 & $99 / 23.1$ & 0.33 \\
$90 / 5.1$ & 0.73 & $90 / 13.1$ & 0.60 \\
$90 / 22.1$ & 0.88 & $90 / 14.1$ & 0.78 \\
$90 / 24.1$ & 0.56 & $90 / 19.1$ & 0.75 \\
$91 / 3.1$ & 1.00 & $91 / 2.2$ & 0.67 \\
$91 / 3.2$ & 0.89 & $92 / 4.1$ & 0.29 \\
$91 / 5.1$ & 0.90 & $\bar{x} \pm \mathrm{s}^{\mathrm{z}}$ & 0.85 \\
$94 / 32.1$ & 0.75 & 0.40 \\
$\bar{x} \pm \mathrm{s}^{\mathrm{z}}$ & $0.78 \pm 0.05$ & $0.55 \pm 0.08$ \\
\hline
\end{tabular}

y Frequency of satRNA-infected plants over total Fny-CMV-infected plants.

${ }^{\mathrm{z}}$ Mean \pm standard error for all necrogenic or nonnecrogenic satRNA variants. 
with Fny-CMV and the set of 10 necrogenic and 10 nonnecrogenic CMV satRNA variants were inoculated into Chenopodium quinoa leaves. For each of the 20 satRNA variants, 10 necrotic local lesions were transferred to 10 small Xanthi-nc tobacco plants and, 2 weeks later, the number of tobacco plants infected by CMV and CMV satRNA was determined. The fraction of tobacco plants infected by CMV that are also infected by CMV satRNA varies for each particular satRNA variant, but, on the whole, is significantly $(P<0.01)$ higher for necrogenic than for nonnecrogenic variants (Table 4).

The results in this section indicate that none of the analyzed fitness components in the satRNA life cycle indicate a disadvantage of necrogenic versus nonnecrogenic satRNA variants, but rather the opposite. Thus, in an attempt to understand the difference in fitness shown by necrogenic and nonnecrogenic variants in nature, we looked at factors related to the interaction between CMV satRNA and its HV.

Quantitative analysis of the interaction of CMV and CMV SatRNA. It is well documented in the literature that the presence of the satRNA results in a depression of the accumulation of CMV to a degree that varies with the isolate of CMV and the variant of satRNA that interact (8). CMV quantification in the experiments described in the previous section, which involved Fny-CMV as a $\mathrm{HV}$, showed that necrogenic satRNA variants, as a whole, caused a significantly bigger depression in CMV accumulation than nonnecrogenic variants (data not shown). To obtain a more representative estimation of this effect, 10 different field isolates of CMV (five of genetic type 1 and five of genetic type 2) were each inoculated into five Rutgers tomato plants alone or in combination with five necrogenic satRNA variants and five nonnecrogenic ones. For each plant, virus particles were purified and quantified by ELISA. Each satRNA variant caused a significant depression in the accumulation of each HV (data not shown), and the degree of that depression varied for each satRNA when all HV were pooled together (Table 5). In addition, when all data were considered, necrogenic satRNA variants caused a significantly larger $(P<0.0001$ in orthogonal comparisons) depression of $\mathrm{HV}$ accumulation than did nonnecrogenic variants (Table 5).

\section{DISCUSSION}

From 1986 to 1992 , an epidemic of tomato necrosis caused by CMV + CMV satRNAs occurred in the tomato-producing coastal areas of eastern Spain, between the provinces of Barcelona to the north and Almería to the south (10). Tomato necrosis first appeared in a small area north of Valencia in 1986, expanded to new areas northward and southward in the following years, and di-

TABLE 5. Accumulation of Cucumber mosaic virus (CMV) particles in tomato leaves after treatment with satellite RNA (satRNA)

\begin{tabular}{lc}
\hline Treatment, satRNA variant & Virus accumulation $^{\mathrm{z}}$ \\
\hline None & $1,124.49 \pm 326.37$ \\
Necrogenic & $170.21 \pm 23.16$ \\
$89 / 15.1$ & $116.88 \pm 16.10$ \\
$89 / 42.1$ & $97.70 \pm 14.77$ \\
$90 / 22.1$ & $97.59 \pm 11.10$ \\
$91 / 5.1$ & $81.50 \pm 10.90$ \\
$94 / 32.1$ & $111.40 \pm 7.33$ \\
$\quad$ Total & \\
Nonnecrogenic & $291.24 \pm 56.21$ \\
89/15.2 & $270.66 \pm 46.31$ \\
89/20.1 & $219.19 \pm 28.03$ \\
$90 / 17.1$ & $358.96 \pm 70.87$ \\
$90 / 19.2$ & $211.20 \pm 40.76$ \\
$91 / 2.2$ & $265.29 \pm 21.75$ \\
Total &
\end{tabular}

${ }^{\mathrm{z}}$ Data are micrograms of virus particles per gram fresh weight in purified virion preparations of systemically infected leaves (mean \pm standard error) when 10 different CMV field isolates were inoculated without satRNA, or with five nonnecrogenic or with five necrogenic satRNA variants. Five plants per treatment were analyzed. Data of CMV isolates are pooled. Except for treatment without satRNA, all considered plants were with satRNA. minished in frequency after 1990 to almost completely disappear after $1991(1,10)$. CMV is endemic in horticultural areas of Spain, but CMV satRNAs had not been reported as associated with isolates of CMV obtained from field-infected vegetable crops until the appearance of tomato necrosis in $1986(10,18)$. Thus, it could be that the epidemic of tomato necrosis reflected an epidemic of CMV satRNA over the CMV population. Alternatively, the tomato necrosis epidemic could reflect the expansion and decrease in the CMV satRNA population of satRNA variants that were necrogenic for tomato. In the field, satRNA variants necrogenic and nonnecrogenic for tomato were found to be present at the same time (10), and CMV satRNAs were found in the CMV population until 1994, after the disappearance of tomato necrosis (1). These two observations supported the second hypothesis above. In this article, we present data that show this was indeed the case. A main result is the significant association between symptoms shown by field tomato plants and the phenotype of the satRNA variants isolated from them. It is also important that CMV satRNA variants necrogenic for tomato were necrogenic, regardless of the strain of $\mathrm{HV}$, and that no effect of $\mathrm{HV}$ on the modulation of the development of necrosis was apparent, a result unlike that in other reports $(27,31)$. Moreover, in mixed infections involving necrogenic and nonnecrogenic satRNAs, tomato necrosis was, in most cases, the dominant phenotype, as reported by other authors (13), so that a relatively minor fraction of necrogenic satRNAs could significantly affect levels of tomato necrosis in the field. Thus, our data indicate that the decrease in tomato necrosis that occurred after 1990 was due to a change in the genetic composition of the satRNA population (i.e., to the evolution of that population toward decreased virulence).

It is often assumed that populations of parasites will evolve toward decreased virulence. Evidence for this assumption derives mostly from the many examples of relatively harmless infections in hosts with which parasites have a long-established relationship, as opposed to the serious impact of the same parasites in new host species. Nevertheless, theoretical support for this assumption has been challenged (21). Well-documented case studies from natural populations are scarce; perhaps the best example is that of myxoma virus infecting rabbits in Australia, whose populations evolved toward intermediate levels of virulence (6). To our knowledge, this work is the first report on the evolution of virulence for a plant viral disease. Other reported epidemics of tomato necrosis caused by CMV satRNAs also resulted in the disappearance of field necrosis after some time $(14,19)$, but no data on the frequency of satRNA variants was given for these events.

It is important to understand which factors determine the observed decrease of virulence of the CMV satRNA population. For this, we carried out a series of experiments aimed at the quantitative estimation of factors in the satRNA life cycle that could affect the relative fitnesses of satRNA variants that were necrogenic and nonnecrogenic for tomato. It was found that necrogenic and nonnecrogenic variants, on the whole, did not differ in their infectivity or in their accumulation levels in tomato leaves and that they represented, on average, the same fraction of virion RNA. Other factors, though, were positively correlated with the higher virulence of necrogenic satRNA variants. In mixed infections, necrogenic satRNAs were favored compared with nonnecrogenic ones, because their accumulation level did not change relative to that in single infections, yet had an effect of depression on the accumulation level of nonnecrogenic variants. Also, necrogenic satRNA variants are more effectively passed into the progeny than were nonnecrogenic variants. This result is in apparent contradiction to the fact that both types of satRNAs constituted, on the average, the same fraction of virion RNA. Nevertheless, the assay of the probability of transmission of satRNA after a single-lesion passage provides an estimate of the probability of satRNA being present in the (minimum) set of particles that must encapsidate CMV RNA1, RNA2, and RNA3, which are needed to start an infection. This probability would be affected by the distribution of satRNA in the particles that encapsidate RNA1, 
RNA2, and RNA3 + RNA4, or in particles that encapsidate only satRNA. That distribution is not known $(16,25)$ and could differ for necrogenic and nonnecrogenic satRNAs. All of these factors, positively correlated with high virulence, would favor necrogenic satRNA variants as compared with nonnecrogenic ones.

However, our results also show that necrogenic satRNA variants cause a more pronounced depression on the accumulation of its $\mathrm{HV}, \mathrm{CMV}$, than did nonnecrogenic ones. Thus, necrogenic satRNAs, in addition to being more virulent to the tomato host plant (i.e., they cause a lethal necrosis versus a stunt, mosaic, and curl, and reduction of leaf lamina) when supported by any CMV isolate, are more virulent, as hyperparasites of $\mathrm{CMV}$, than are nonnecrogenic ones. This virulence-related effect on the accumulation of the HV can have an important impact on the efficiency of aphid transmission of CMV + CMV satRNAs, because it has recently been shown that aphid transmissibility of CMV and CMV+satRNA depends primarily on the concentration of CMV particles in tomato leaf tissues (F. Escriu, K. L. Perry, and F. García-Arenal, unpublished data).

It must be considered that the interplay between the above-analyzed fitness components could be affected by factors external to CMV and CMV satRNA. An important environmental factor could be the temperature. High temperatures affect the accumulation of necrogenic and nonnecrogenic satRNAs differently, and this is dependent on the strain of $\mathrm{HV}(11,30)$. The rise of temperatures early in July resulted in a stop of the progress of tomato necrosis in the field (10), which could reflect such an interaction between temperature and satRNA accumulation. Also, the relative accumulation of satRNA variants necrogenic and nonnecrogenic for tomato could be different in other host plant species, because it has already been shown that satRNA accumulation depends on the trilateral interaction: strain of CMV/strain of satRNA/species of host plant (12). A host species effect would be of epidemiological relevance in the life cycle of CMV satRNA. The analysis of these other factors is out of the scope of this article, which is limited to the analysis of the interplay between factors related to virulence and factors related to transmission. Theoretical considerations show that the evolution of virulence in populations of parasites can follow many paths and that it mainly depends on trade-offs between virulence and transmission, both of which are rarely independent components (20). Trade-offs between the analyzed factors that favor accumulation and passage upon CMV transmission of necrogenic satRNAs and those that would result in less-efficient aphid transmission could explain the observed evolution of virulence of satRNA populations in eastern Spain. Nonnecrogenic CMV satRNAs also cause a reduction in the accumulation levels of CMV; therefore, the same factors could also be involved in the disappearance of CMV satRNAs from Spanish populations of CMV after 1994 (1).

\section{ACKNOWLEDGMENTS}

F. Escriu was supported by fellowships of Plan de Formación de Personal Investigador de la Comunidad Autónoma de Madrid and of Colaboración en Proyectos de Investigación Científica y Técnica de la Universidad Politécnica de Madrid, Spain, during this study. The work was supported, in part, by grant AGF97-0918, CICYT, Spain.

\section{LITERATURE CITED}

1. Alonso-Prados, J. L., Aranda, M. A., Malpica, J. M., García-Arenal, F., and Fraile, A. 1998. Satellite RNA of cucumber mosaic cucumovirus spreads epidemically in natural populations of its helper virus. Phytopathology 88:520-524.

2. Aranda, M. A., Fraile, A., Dopazo, J., Malpica, J. M., and García-Arenal, F. 1997. Contribution of mutation and RNA recombination to the evolution of a plant pathogenic RNA. J. Mol. Evol. 44:81-88.

3. Aranda, M. A., Fraile, A., and García-Arenal, F. 1993. Genetic variability and evolution of the satellite RNA of cucumber mosaic virus during natural epidemics. J. Virol. 67:5896-5901.

4. Bernal, J. J., and García-Arenal, F. 1994. Analysis of the determinants of the satellite RNA of cucumber mosaic cucumovirus for high accumulation in squash. Virology 205:262-268.
5. Ebert, D. 1998. Experimental evolution of parasites. Science 282:1432-1435.

6. Fenner, F. 1995. Classical studies of virus evolution. Pages 13-30 in: Molecular Basis of Virus Evolution. A. Gibbs, C. H. Calisher, and F. García-Arenal, eds. Cambridge University Press, Cambridge.

7. Fraile, A., Alonso-Prados, J. L., Aranda, M. A., Bernal, J. J., Malpica, J. M., and García-Arenal, F. 1997. Genetic exchange by recombination or reassortment is infrequent in natural populations of a tripartite RNA plant virus. J. Virol. 71:934-940.

8. García-Arenal, F., and Palukaitis, P. 1999. Structure and functional relationships of satellite RNAs of cucumber mosaic virus. Pages 37-63 in: Current Topics in Microbiology and Immunology. Vol. 239: Satellites and Defective Viral RNAs. P. K. Vogt and A. O. Jackson, eds. SpringerVerlag, Berlin.

9. Gerlach, W. L., and Bedbrook, J. R.1979. Cloning and characterization of ribosomal RNA genes from wheat and barley. Nucleic Acids Res. 7:1869-1885.

10. Jordá, C., Alfaro, A., Aranda, M. A., Moriones, E., and García-Arenal, F. 1992. Epidemic of cucumber mosaic virus plus satellite RNA in tomatoes in eastern Spain. Plant Dis. 76:363-366.

11. Kaper, J. M., Geletka, L. M., Wu, G. S., and Tousignant, M. E. 1995. Effect of temperature on cucumber mosaic virus satellite-induced lethal tomato necrosis is helper virus strain dependent. Arch. Virol. 140:65-74.

12. Kaper, J. M., and Tousignant, M. E. 1977. Cucumber mosaic virus-associated RNA5. I. Role of host plant and helper virus in determining the amount of associated RNAs with virions. Virology 80:186-195.

13. Kaper, J. M., Tousignant, M. E., and Steen, M. T. 1988. Cucumber mosaic virus-associated RNA5. XI. Comparison of 14 CARNA 5 variants relates ability to induce tomato necrosis to a conserved nucleotide sequence. Virology 163:284-292.

14. Kosaka, Y., Hanada, K., Fukinushi, T., and Tochihara, H. 1989. Cucumber mosaic virus isolate causing tomato necrosis disease in Kyoto Prefecture. Ann. Phytopathol. Soc. Jpn. 55:229-232.

15. Lennard, P. R. 1990. Image analysis for all. Nature 347:103-104.

16. Lot, H., and Kaper, J. M. 1976. Further studies on the RNA component distribution among the nucleoproteins of cucumber mosaic virus. Virology $74: 223-226$.

17. Lot, H., Marrou, J., Quiot, J. B., and Esvan, C. 1972. Contribution à l'étude du virus de la mosaïque du concombre (CMV). Méthode de purification rapide du virus. Ann. Phytopathol. 4:25-38.

18. Luis-Arteaga, M., Rodríguez-Cerezo, E., Maestro, C., and García-Arenal, F. 1988. Detection and characterization of an isolate of cucumber mosaic virus infecting borage (Borago officinalis L.) in Spain. Plant Dis. 72:265-267.

19. Marrou, J., Duteil, M., Lot, H., and Clerjeau, H. 1973. La nécrose de la tomate: Une grave virose des tomates cultivées en plein champ. Pepinieres Hortic. Maraichere 137:37-41.

20. May, R. M. 1995. The co-evolutionary dynamics of viruses and their hosts. Pages 192-212 in: Molecular Basis of Virus Evolution. A. Gibbs, C. H. Calisher, and F. García-Arenal, eds. Cambridge University Press, Cambridge.

21. May, R. M., and Anderson, R. M. 1983. Epidemiology and genetics in the coevolution of parasites and hosts. Proc. Royal Soc. Lond. B. 219: 281-313.

22. Moriones, E., Díaz, I., Rodríguez-Cerezo, E., Fraile, A., and García-Arenal, F. 1992. Differential interactions among strains of tomato aspermy virus and satellite RNAs of cucumber mosaic virus. Virology 186:475-480.

23. Owen, J., and Palukaitis, P. 1988. Characterization of cucumber mosaic virus. I. Molecular heterogeneity mapping of RNA3 in eight strains. Virology 166:495-502.

24. Palukaitis, P., and Roossinck, M. J. 1996. Spontaneous change of a bening satellite RNA of cucumber mosaic virus to a pathogenic variant. Nat. Biotechnol. 14:1264-1268.

25. Palukaitis, P., Roossinck, M. J., Dietzgen, R. G., and Francki, R. I. B. 1992. Cucumber mosaic virus. Adv. Virus Res. 41:281-348.

26. Sambrook, J., Fritsch, E. F., and Maniatis, T. 1989. Molecular Cloning: A Laboratory Manual. Cold Spring Harbor Laboratory, Cold Spring Harbor, NY.

27. Sleat, D. E., Zhang, L., and Palukaitis, P. 1994. Mapping determinants within cucumber mosaic virus and its satellite RNA for the induction of necrosis in tomato plants. Mol. Plant-Microbe Interact. 7:189-195.

28. Sokal, R. R., and Rohlf, F. J. 1981. Biometry. 2nd ed. W. H. Freeman, New York.

29. Thompson, J. R., and García-Arenal, F. 1998. The bundle sheath-phloem interface of Cucumis sativus is a boundary to systemic infection by tomato aspermy virus. Mol. Plant-Microbe. Interact. 11:109-114.

30. White, J. L., Tousignant, M. E., Geletka, L. M., and Kaper, J. M. 1995. The replication of a necrogenic cucumber mosaic virus satellite is temperature-sensitive in tomato. Arch. Virol 140:53-63.

31. Wu, G., and Kaper, J. M. 1992. Widely separated sequence elements within cucumber mosaic virus satellites contribute to their ability to induce lethal tomato necrosis. J. Gen. Virol. 73:2805-2812. 